个顶点的图，且对任何 $x y \notin E(c i)$ 总有 $d(x)+d(y)$ $\geqslant n$, 则线图 $L(G)$ 是 Hanilton 图.

1984 年, Clark 得到更强的Ore 类条件,

定理（Clark） 如果 $G$ 是有 $n \geqslant 6$ 个顶点的 图,且对任何 $x y \notin E(G)$ 总有 $d(x)+d(y) \geqslant n-1-$ $p(n)$, 则线图 $L(G)$ 是 Hmailton 图, 这里

$$
p(n)= \begin{cases}0 & n \text { 是偶数. } \\ 1 & n \text { 是奇数. }\end{cases}
$$

在本文中, 我们得到下述更一般结果.

定理 1 如果连通图 $G$ 是边三角形的, 则线 图 $L(G)$ 是顶点泛圈图.

推论 $n$ 个顶点的图 $G$, 若对任何 $x y \notin E(G)$, 总有 $\boldsymbol{d}(x)+d(y) \geqslant n+1$, 则线图 $L(G)$ 是顶点
泛圈图.

定理 2 如果 $G$ 是有 $n \geqslant 6$ 个顶点的图, 且 对任何 $x y \notin E(G)$, 总有 $d(x)+d(y) n \geqslant n-1-p(n)$, 则线图 $L(G)$ 是泛圈图. 事实上, 线图 $L(G)$ 的每 个顶点都含在某个k圈上, 这里,$\leqslant k \leqslant|V(G)|$ 和 $k \neq 4$.

最近, 占四明得到结果: 每个 4 连通图的线图 是 Hamilton 图. 事实上, 我们有

定理 3 如果 $G$ 是 4 边连通图, 则线图 $L(G)$ 是 Hamilton

施容华

(青海师范大学数学系, 西宁)

\title{
度序列已给定的部分标定图的重构问题
}

设 $G=(V, E)$ 是简单、无向的 $p$ 阶部分标定图， $v=\left\{v_{1}, \cdots, v_{p}\right\}, p \geqslant 3$. 设 $u, v \in V, X, Y \subset V$. 记 $N_{Y}$ (v) 为顶点 $v$ 在 $Y$ 中的邻集, $d_{Y}(v)=\left|N_{Y}(v)\right|$ 为 $v$ 关于 $Y$ 的度，为 $v$ 关于 $Y$ 的邻揬向量，它的第 $i$ 个分量为 0 (或 1), 对应于 $v$ 与 $Y$ 的第 $i$ 个顶点不邻 接(或邻接). 若 $d_{Y}(u)=d_{Y}(v)$, 称 $u, v$ 关于 $Y$ 等 度; 若 $u, v \notin Y$ ，且 $\boldsymbol{u}(Y)=\boldsymbol{v}(Y)$ ，称 $u, v$ 关于 $Y$ 等邻; 若 $u, v$ 关于 $X$ 等度, 关于 $Y$ 等邻, 称 $u, v^{2}$ 关于 $(X, Y)$ 等色. 显然， $X$ 上的等度, 等邻和等色关系 都是等价关系, 由此所划分的等价类分别称为等度 集,等邻集和管色集.

定义 1 在主子图 $G_{j}$ 中, $U_{j}$ 为朱标定集, $L$ 为标定集. 设 $U_{i}=\sum_{i=1}^{m} X_{i}, X_{i}(1 \leqslant i \leqslant m)$ 是 $U_{j}$ 关 于 $L$ 的等邻集. 若 $u, v \in U$; 关于 $L$ 等邻, 且存在 $G_{i}$ 的一个自同构 $\alpha$ 满足 $1^{\circ} \alpha\left(u^{\prime}\right)=u, \quad \alpha(u)=v$; $2^{\circ} \alpha\left(X_{i}\right)=X_{i}, \quad 1 \leqslant i \leqslant m ; 3^{c} \alpha(L)=L$, 则称 $u$, $v v$ 在 $G$; 中关于 $L$ 等邻相似, 记为 $\stackrel{L}{L} v^{\prime}$. 显然, 等 邻相似关系也是等价关系.

定义 2 设 $S$ 在主子图 $C_{j}$ 中可标记. 若 $N_{s}$ $\left(v_{i}\right)$ 在 $G_{j}$ 中也可标记, 则称主子图 $G_{i}$ 具有性质 $\mathscr{B}(S)$, 记为 $G_{j} \in \mathscr{B}(S)$. 特别, 把 $G_{j} \in \mathscr{B}\left(v^{\prime}{ }_{i}\right)$ 简记 为 $G_{i} \in \mathscr{B}$.

引理 1 给出 $p$ 阶图 $G$ 的前 $n-1$ 个主子 图 $(n \geqslant 4)$, 其中 $v_{1}, \cdots, v_{n}$ 末标定, ${ }^{\prime}{ }_{n+1}, \cdots, v_{p}$ 标 定, 则 $\boldsymbol{v}_{i}(L)$ 确定 $(1 \leqslant i \leqslant n)$.

第 2 期
引理 2 给定 $p$ 阶图 $G$ 的度序列 $d_{i}(1 \leqslant i \leqslant$ $p)$, 设 $L$ 是主子图 $G_{i}$ 的标定集, 则 $\boldsymbol{v}_{j}(L)$ 确定.

引理 3 在主子图 $G_{j}$ 中, $\left\{X_{i}(0 \leqslant i \leqslant m)\right\}$ 是 未标定集 $U_{j}$ 的一个分划, $X_{i}$ 的各点均关于标定 集 $L$ 等邻. 记 $X_{0}=\left\{v_{x}, v_{y}, \cdots, v_{w}\right\}$. 若满足 $1^{\circ} X_{i}$ $(1 \leqslant i \leqslant n)$ 中各点关于 $\left\{v_{j}\right\}$ 等邻; $2^{\circ} v_{x} \sim v_{y}, L \ldots$ $L_{u^{\prime}{ }_{w}}^{L} ; 3^{\circ}{ }^{\prime}{ }_{i}$ 与 $V-X_{0}$ 的邻接关系已确定; $4^{\circ} d_{X 0}\left(v_{i}\right)$ 确定, 则分别按满足 $4^{\circ}$ 的各种情形由 $G_{i}$ 所得重构 图相互同构.

引理 4 给出主子图 $G_{i}$, 若 $G_{i} \in \mathscr{B}$, 且 $\boldsymbol{v}_{i}$ (L) 确定, $L$ 是标定集,则 $G$ 可由 $G_{i}$ 重构.

引理 5 设 $U_{i}$ 是 $G_{i}$ 的未标分集, $S, T \subset$ $U_{i}$. 若 $G_{i} \in \mathscr{B}(S), G_{j} \in \mathscr{F}\left(T^{\prime}\right)$, 则 $G_{j} \in \mathscr{B}(S U T)$.

引理 6 给出 $G$ 的三个主子图 $G_{5}, G_{6}, C_{7}$. 设 $U=\left\{v_{1}, v_{2} 、 v_{3}, v_{4}\right\}$ 是未标定集, $T \subset U v_{5}, v_{6} v_{7}$ 在 $G_{i}(5 \leqslant i \leqslant 7)$ 中均已标定, $T$ 在 $G_{i}(5 \leqslant i \leqslant 7)$ 中 已标记. 记 $d_{T}\left(v_{i}\right)=l_{i}(5 \leqslant i \leqslant 7), \quad 0 \leqslant l_{i} \leqslant|T|$, 则 $1^{\circ}$ 当 $l_{i}=0$ 或 $|T|$ 时, $G_{i} \in \mathscr{B}(T) ; 2^{\circ}$ 当 $|T|$ $=1$ 时, $G_{i} \in \mathscr{B}(T), 5 \leqslant i \leqslant 7 ; 3^{\circ}$ 当 $|T|=2$ 且 $l_{i}=1(5 \leqslant i \leqslant 7)$ 村, $G_{j} \in \mathscr{B}(T), 5 \leqslant j \leqslant 7 ; 4^{\circ}$ 当 $|T|$ $=3$ 且 $l_{i}=1$ 或 $2(5 \leqslant i \leqslant 7)$ 时, 至少有二个 主子图具有性质 $\mathscr{B}(T)$.

利用等邻和等色关系, 由上述引理我们证明了 以下定理:

定理设 $p$ 阶部分标定图 $G$ 的度序列 $d_{i}(1 \leqslant$ $i \leqslant p)$ 已给定, 其中前 $n$ 个顶点末标定 $(n \geqslant 3)$, 其 
当 $n=3$ 或 | 时, $G$ 可由所给出的主子图重构.

我们猜想, 上述定理对任何正整数 $n \geqslant 3$ 均成立.
叶秀明

(上海科学技术大学)
鄂学才

(北京工业大学)

\section{Lamyerti 算子的一些性质}

余顶点均已标定. 给出 $G$ 的任意 $n-1$ 主子图,则

$E$ 是相对一致完备的向量格， $T$ 是 $E$ 上的格同 态, $\sigma_{p}(T)$ 代表 $T$ 的点谱. $\lambda 、 \mu \in \sigma_{p}(T) \backslash\{0\}, T x$ $=\lambda x, T y=\mu y, x 、 y \neq 0$. W. A. Wickstead 证明了 如 $|\lambda| \neq|\mu|$. $\lambda$ 不是 $\sigma_{p}(T)$ 的极限点, 则 $|x| \wedge|y|$ $=0$, 亦即 $x 、 y$ 不交. 并由此给出了紧格同态的谱 分解, 当 $E$ 是具有序连续范数的 Banach 格, 且 $T^{\prime}$ 也 是格同态时. 这里把这些结果推到了 Lamperti 算 子. 我们有

定理 $1 E$ 是相对一致完备的向量格, $T$ 是 $E$ 上的 Lamperti 算子, $\lambda 、 \mu \in \sigma_{p}(T) \backslash\{0\}, T x=$ $\lambda x, T y=\mu y$. 若 $|x| \wedge|y| \neq 0$, 则

$$
\Gamma_{\lambda \mu} \subset \sigma_{p}(T) \text {. }
$$

其中

$$
\Gamma_{\lambda \mu}=\left\{\alpha \lambda \mid \alpha \in\left[1, \frac{|\mu|}{|\lambda|}\right]\right\} \cup\left\{\beta \mu \mid \beta \in\left[\frac{|\lambda|}{|\mu|}, 1\right]\right\}
$$

当 $|\lambda| \leqslant|\mu|$ （若 $|\lambda| \geqslant|\mu|$, 交换一下 $\lambda, \mu$ 的位 置).

定理 $2 E$ 是具有序连续范数的 Banach 格, $T$ 是 $E$ 上紧 Lamperti 算子, 且 $T$, 也是 Lamperti 算子,则

$$
E=\left(\underset{r \in A}{\oplus_{r}}\right) \oplus K, “ \oplus ” \text { 代表序直和. }
$$

其中 $\Lambda=|\sigma(T)| \backslash\{0\}, H_{r} 、 K$ 都是 $T$ 的不变理想,
$H_{r}$, 是有限维. $\left.T\right|_{K}$ 是拟幂零的; $\sigma\left(T \mid H_{r}\right)=\{\lambda \in \sigma$ $(T)\}|\lambda|=r\}$.

其次, $E, F$ 是 Banach 格, $T: E \longrightarrow F$, 如果 $|T|$ 存在, 那么 $|T|$ 与 $T$ 紧性之间的关系问题一直是 一个基本问题. 这里, 对 Lamperti 算子有下述结 果.

定理 $3 E 、 F$ 是 Banach 格, $T: E \longrightarrow F$ 是 Lamperti 算子. 则下列分解成立:
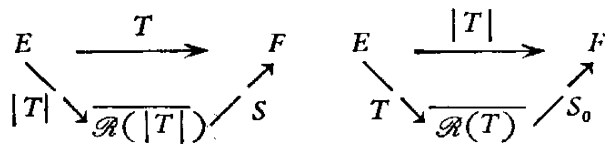

其中, $S 、 S_{0}$ 是等距;且 $S S_{0}=I \mathscr{R}_{(T)}, S_{0} S=I \mathscr{R}_{(|T|}$. 因 此, $T$ 紧的充要条件是 $|T|$ 紧; $T$ 弱紧的充要条件是 $|T|$ 弱紧。

最后,与 W. Arendt 的结果 ( $T$ 是 Lamperti 算 子, 则 $|\sigma(T)|=\sigma(|T|) \cap[0, \infty))$ 相仿, 我们有

定理 $4 T$ 是 Banach 格 $E$ 上的 Lamperti 算 子, 则

$$
\left|\sigma_{A}(T)\right|=\sigma_{A}(|T|) \cap[0, \infty)
$$

其中 $\sigma_{A}(T) 、 \sigma_{A}(|T|)$ 分别代表 $T 、|T|$ 的逼近点 谱.

\section{陈滋利}

（陕西师范大学数学系,西安）

\section{Banach 空间的一个 性 质}

对矢值测度 $\mu \in b a(S, \Sigma, X)$ 的分解 $\mu=\mu_{I}+$ $\mu_{I I}$ 的意义如同前文 (数学杂志, 3(1984), 285-2 92). 今记 $b a_{I}(S, \Sigma, X)=\left\{\mu_{I}: \mu \in b a(S, \Sigma, X), b_{x_{I I}}\right.$ $(S, \Sigma, X)=\left\{\mu_{I I}: \mu \in b a(S, \Sigma, X)\right\}$.

定理 1 若 $\Sigma$ 是集 $S$ 的一些子集作成的代 数,含有 $s$ 的一切有穷子集, $X$ 是 Banach 空间,则有 界变差测度空间 $b a(S, \Sigma, X)$ 有分解 $b a(S, \Sigma, X)=$ $b a_{I}(S, \Sigma, X)+b a_{I I}(S, \Sigma, X)$.

定理 2 若 $x$ 是 Banach 空间, $U_{X}$ 是 $x$ 的闭
单位球,则对每个 $x \in X$ 有 $\mu \in b a_{I}\left(U_{X}\right)$ 适合

$$
x^{*}(x)=\int_{U_{X}} x^{*}(y) \mu(d y), \forall x^{*} \in X^{*},
$$

并且每个 $\mu \in b a_{I}\left(U_{X}\right)$ 则通过上式表示某个 $x \in X$. 此结果不能被推广到赋范空间的情形，例如， $l_{1}$ 的椆密真子空间 $\varphi$ 不具备定理 2 所主张的性质.

\section{李容录}

(哈尔滨工业大学) 